\title{
L'immigrazione al tempo della pandemia: nuove difficoltà, scoperte impreviste, opportunità insperate
}

\author{
Maurizio Ambrosini
}

La pandemia da COVID-19 si è intrecciata con le questioni dell'immigrazione e dell'asilo in diversi modi. Nel momento in cui questo editoriale viene redatto (fine maggio 2020) l'emergenza non è finita, le sue conseguenze socio-economiche rimangono incerte nelle loro proporzioni, l'efficacia delle misure di protezione e rilancio varate dal governo è altrettanto sconosciuta.

Sul piano scientifico o anche soltanto conoscitivo disponiamo al momento di pochi dati e pochissima letteratura per discutere dell'impatto del COVID-19 sulle popolazioni immigrate. L'aspetto più rilevante riguarda il faticoso e contrastato varo di una procedura parziale di emersione, da parte di un sistema politico che nelle elezioni del 2018 aveva chiesto e ottenuto un mandato programmaticamente ostile a ogni apertura nei confronti di immigrati e rifugiati. A questo aspetto dedicherò una particolare attenzione, ma tenendo conto di altri elementi emersi in questo drammatico periodo: lo sgonfiamento della retorica dell'invasione e l'avvento della sinofobia; l'incidenza delle chiusure sulle precarie condizioni economiche di molti immigrati; il contributo degli immigrati nei lavori essenziali, e in modo particolare nella sanità.

\section{Scesa la febbre dell'invasione, sinofobia e nuove chiusure}

Quasi di colpo, l'emergenza sanitaria ha scacciato dai mass-media, dalle reti social e dalle conversazioni quotidiane l'ossessione dell'invasione. Il leader della Lega Salvini, suo massimo propugnatore, ha persino perso un po' di terreno nei sondaggi. Certo, sono diminuiti ancora gli sbarchi, già da tempo in realtà pochissimi. Ma soprattutto l'opinione pubblica ha scoperto altri e ben più consistenti motivi di preoccupazione, altri fenomeni su cui indirizzare le proprie ansie e paure.

L'invasione, come abbiamo sempre sostenuto su questa rivista e in tutte le sedi possibili, non è mai esistita (Ambrosini, 2018; 2020). Finalmente è sparita dai titoli di testa e (quasi) dalle invettive in rete. Non che responsabili e seguaci di questa allucinazione di massa abbiano ammesso l'errore, sviluppando qualche seria riflessione autocritica. Semplicemente, hanno voltato pagina, trovando altra materia su cui esercitare da parte di alcuni una comunicazione drammatizzante, a forti tinte emozionali e con inclinazioni paranoidi; da parte dei più, e in modo particolare dei media più diffusi e influenti, un corrivo adeguamento alla visione prevalente, alle pulsioni del pubblico o almeno della sua componente più rumorosa, senza un sufficiente sforzo di reazione critica.

Il cambio di allarme non ha però giovato agli immigrati in modo univoco. Abbiamo assistito, soprattutto nelle prime fasi, a fenomeni inquietanti di ripulsa ed emarginazione nei confronti di persone di origine cinese e a volte di altri asiatici; nonché di nuovi pretesti di chiusura nei confronti dei richiedenti asilo provenienti dall'Africa.

La paura che gli stranieri (specie se poveri) diffondano malattie è antica e radicata. L'argomento era già stato invocato negli scorsi anni nei confronti dei rifugiati africani, da alcuni additati come portatori di Ebola, da molti altri tenuti alla lontana anche per presunti rischi sanitari. Alcuni influenti protagonisti dei social avevano diffuso notizie di numerosi casi di TBC e altre malattie. Molti ricorderanno gli agenti di polizia accoglierli con guanti e mascherine. Ma non c'è stata notizia di vere 
o presunte epidemie che non abbia sollevato la richiesta di chiusura delle frontiere verso rifugiati e immigrati dal Sud del mondo.

In termini generali, le ricerche sull'argomento, come quelle condotte dalla Società Italiana di Medicina delle Migrazioni (SIMM), segnalano invece che i migranti sono selezionati alla partenza da un punto di vista sanitario: ben raramente famiglie e comunità investono i propri risparmi o s'indebitano per far partire persone malate. Si forma così un effetto iniziale definito "migrante sano" (Colasanti, Geraci e Pittau 1991). Sono semmai le condizioni di lavoro e di vita dopo l'arrivo a intaccare la salute degli immigrati (Molli 2018).

L'ondata sinofoba conseguente alle prime notizie sulla diffusione del COVID 19 si è rivelata particolarmente incresciosa, tanto da attirare l'attenzione dei mass-media internazionali ${ }^{1}$, perché ha investito cittadini cinesi e naturalizzati residenti qui da anni, nonché attività commerciali, ristoranti, bambini che frequentano le scuole italiane, piccoli calciatori: tutte persone e famiglie che non avevano nessun rapporto con la città di Wuhan e la provincia di Hubei, epicentro dell'epidemia. Il presunto allarme sanitario ha giustificato la discriminazione anche dei più piccoli e indifesi.

Queste reazioni scomposte hanno rivelato alcuni aspetti preoccupanti del nostro tessuto sociale. Se non è giusto affermare che il popolo italiano sia razzista, è altrettanto sbagliato rifugiarsi nell'idea consolatoria secondo cui "gli italiani non sono razzisti"”. Il razzismo attuale, non potendo più fare appello a presunte ragioni "scientifiche", cerca di volta in volta argomenti apparentemente razionali per sostenere la necessità di cacciare, discriminare o emarginare le proprie vittime (cfr, per un approfondimento del tema, Colombo, 2020; per il caso francese Primon, 2019): può essere la disoccupazione, o il terrorismo, o la sicurezza, da ultimo il Covid-19.

Restrizioni più mirate ed effettive si sono abbattute invece sulle persone entrate dal mare o dai confini orientali come richiedenti asilo. Sono partite subito campagne per isolarli o per vietare loro l'ingresso, mentre ancora si auspicava l'arrivo di turisti stranieri, e ora a emergenza attenuata si torna a invocarli. L'idea che i viaggi internazionali di alcuni siano innocui e gli spostamenti di altri siano perniciosi contiene qualcosa di irrazionale, da pensiero magico. Anche se per una sorta di riflesso condizionato continuiamo a guardare con sospetto i migranti poveri, a evitarli o a bloccarli prima che sbarchino, il temibile contagio si è diffuso in Europa e attraverso l'Atlantico mediante ogni sorta di contatto interpersonale. Viaggi d'affari, turismo, visite ai familiari all'estero comportano una circolazione di persone molto maggiore delle migrazioni. In tempi normali nessuno ci fa caso, ma anche nell'emergenza attuale si è visto all'opera un doppiopesismo stridente in materia di precauzioni. Istintivamente, il pericolo è associato alla mobilità dei poveri.

Così, in aprile, in prossimità della Pasqua, il governo ha assunto una decisione drastica: giacché è in corso l'epidemia del COVID-19, l'Italia non ha porti sicuri da offrire, quindi sono vietati gli sbarchi delle persone salvate in mare dalle navi delle ONG fino al 31 luglio. Non la quarantena, come già avvenuto, e come tuttora avviene nei confronti dei richiedenti asilo che arrivano spontaneamente sulle nostre coste. Proprio il divieto di sbarco, con l'eventuale respingimento verso la Libia.

I giornali vicini al centro-destra e ostili ai rifugiati hanno esultato: ci voleva un'epidemia per chiudere finalmente i porti. A essere indulgenti, si può pensare a un tentativo maldestro di riprovare ad addossare gli oneri dell'accoglienza ai nostri partner europei. Tenendo conto che Francia e Spagna possono accampare le nostre stesse motivazioni, la nave Alan Kurdi ha fatto rotta verso la Germania, di cui inalberava la bandiera. È esattamente quanto auspicava il governo Conte 1, con il suo

\footnotetext{
${ }^{1}$ https://www.bbc.com/news/world-asia-51456056, consultato il 27 maggio 2020

${ }^{2}$ Per una riflessione sull'eurocentrismo e il suo superamento: Rebughini e Leonini 2019
} 
ingombrante ministro degli Interni, e veniva allora contestato dall'opposizione di sinistra: quei giorni supplementari in mare, quella convivenza forzata in condizioni deplorevoli, quella permanenza su navi non dotate di servizi adeguati, erano le conseguenze di una politica condannata allora come disumana. Si invocava compassione almeno per i più fragili, minorenni, donne, malati. Con la decisione di aprile quello stesso allungamento del viaggio è stato imposto ai migranti e forse anche celebrato come un successo politico: una trovata vincente del genio italico.

La vicenda sembra confermare quanto osservato da Triandafillydou (2020) a livello internazionale: la pandemia da COVID-19 sembra rinforzare ancora di più il trend di securitizzazione dei confini e di ripiegamento nella sovranità nazionale, nello sforzo di proteggere il gruppo degli inclusi (i cittadini nazionali) dagli outsiders, migranti e rifugiati, percepiti come una minaccia per il benessere nazionale. La crisi da Covid 19 sarebbe "un anello aggiuntivo di questa catena della securitizzazione" (ibid.:1). La solidarietà interna e l'obbligo degli Stati di proteggere i propri cittadini ha rafforzato la contrapposizione verso persone vulnerabili provenienti dall'esterno, nonostante gli appelli di varie ONG e altri attori non governativi.

\section{Immigrati poveri, immigrati essenziali}

Il confinamento ha colpito molti lavoratori, in modo particolare quelli autonomi, precari, non garantiti, sommersi. Ha anche pesato sulle modalità di sopravvivenza dei soggetti marginali, dai parcheggiatori abusivi, ai suonatori ambulanti, ai mendicanti. Tra di loro è elevata la percentuale d'immigrati, che si sono trovati immediatamente esposti all'impatto economico della pandemia. Spesso anche non coperti dagli ammortizzatori sociali.

L'incidenza della povertà assoluta già nel 2018 secondo l'ISTAT raggiungeva il 30,3\% tra i cittadini stranieri, contro il $6,4 \%$ tra i cittadini italiani. In cifre, oltre 1,5 milioni di persone, 567.000 nuclei familiari, pari al 31,3\% delle famiglie che versano in povertà assoluta ${ }^{3}$. Difficile credere che la situazione sia migliorata.

Pur essendo risalita l'occupazione degli immigrati, dopo il crollo del $2008(+760.000$ occupati in dieci anni, con un'incidenza sull'occupazione complessiva passata da meno del $7 \%$ a oltre il $10 \%)^{4}$, gravi sacche di povertà non erano ancora state riassorbite. La contrazione dell'attività economica dovuta al confinamento ha pesato su questo contesto di fragilità. Per citare un esempio, oltre la metà dei commercianti ambulanti sono nati all'estero: la chiusura dei mercati all'aperto li ha improvvisamente privati di lavoro e reddito. Come gli italiani, certo, ma a partire da condizioni economiche più disagiate, con poche risorse da parte e in molti casi avendo contratto debiti per l'avvio dell'attività

L'impoverimento degli immigrati ha poi implicazioni transnazionali sul versante delle rimesse. Secondo le prime stime della Banca Mondiale, si prevede per il 2020 un calo di circa il 20\%: in valori assoluti, da 554 miliardi di dollari a 445. Ne saranno colpite le famiglie rimaste in patria, che fanno affidamento sulle rimesse per accedere a cibo migliore, abitazioni più confortevoli, accesso all'istruzione, alla sanità e ai consumi, nonché economie locali e nazionali che contano sulle rimesse degli emigranti per rimediare alla fragilità dei fattori produttivi interni (sul ruolo delle rimesse nelle famiglie transnazionali: Ambrosini, 2019). Sommandosi al crollo del prezzo del petrolio e delle

\footnotetext{
${ }^{3}$ www.integrazionemigranti.gov.it/Attualita/Notizie/Pagine/Povertà-assoluta.aspx. Scaricato il 25 maggio 2020

${ }^{4}$ Faccio qui riferimento agli annuali rapporti sul mercato del lavoro immigrato pubblicati dal Ministero del lavoro. In bibliografia ho citato l'ultimo: Ministero del lavoro 2019.
} 
materie prime, nonché alla sospensione del movimento turistico, la pandemia sta infliggendo al Sud del mondo un colpo economico disastroso (Peloso, 2020).

Il caso che più ha fatto breccia nel discorso pubblico è stato quello delle collaboratrici e assistenti familiari, dette badanti, anche a motivo del loro stretto legame con la tenuta dei precari equilibri delle famiglie per cui lavorano ${ }^{5}$. Secondo l'INPS, a fine 2018 si trattava di 859.233 persone regolarmente registrate, in sette casi su dieci straniere, per 1'83\% donne (Ministero del Lavoro, 2019). Arrivano da tutto il mondo, con cinque paesi in evidenza come fornitori di questi aiuti spesso provvidenziali: Ucraina (21,9\%), Filippine (16,7\%), Moldova (10,1\%), Perù (7,0\%) e Sri Lanka (6,8\%). Fin qui i dati ufficiali. Secondo alcune fonti (Assindatcolf) sarebbero addirittura 2 milioni, 1,15 milioni quindi lavorerebbero in nero (Zini 2019).

La stretta dovuta al coronavirus ha comportato diversi effetti sulle loro già difficili condizioni di lavoro e di vita. Quante lavoravano a ore, magari presso più famiglie, sono state generalmente sospese dal lavoro. Nel primo periodo non erano coperte dagli ammortizzatori sociali, nemmeno dalla cassa integrazione in deroga che tutela i lavoratori delle piccolissime imprese. Niente lavoro, niente stipendio, salvo la generosità di qualche datore. Si può immaginare l'effetto su famiglie già in lotta quotidiana con la povertà, oppure sui figli rimasti al paese di origine accuditi dai nonni o da altri parenti. A maggio il governo ha rimediato a questa emergenza concedendo un assegno di 1.000 euro a copertura parziale di due mesi di sospensione dal lavoro.

Quando le assistenti familiari si occupavano di anziani fragili, in regime di convivenza, si sono trovate minacciate dallo stesso problema: i familiari rimasti a casa, nell'incertezza sui redditi presenti e futuri, potevano decidere di risparmiare sulle spese facendosi carico direttamente dell'assistenza ai congiunti.

Se invece sono riuscite a mantenere il posto di lavoro, si sono trovate anzitutto esposte a un virus molto minaccioso per gli anziani. La loro formazione ad affrontare il contagio, la dotazione di dispositivi di protezione, la capacità di gestire adeguatamente lo spazio domestico, sono nelle mani delle famiglie datrici di lavoro, che a loro volta non hanno una preparazione tecnica per assicurare la prevenzione sanitaria. V'è da chiedersi se qualcuno si sia posto il problema, se siano stati attivati servizi di consulenza a distanza, se siano stato organizzato l'approvvigionamento di mascherine, guanti, disinfettanti e quant'altro servirebbe. Non sappiamo neanche quante assistenti familiari abbiano contratto il virus. Anche a loro spetterebbe una menzione, quando giustamente vengono ricordati i lavoratori che si prodigano per assicurare assistenza a chi ne ha bisogno.

Pure quando non corrono rischi immediati, il blocco delle uscite e delle interazioni sociali le ha private poi di quel minimo di respiro e di contatti col mondo esterno che offrono una valvola di sfogo a chi convive con persone molto anziane e in declino. La reclusione è diventata totale. Con quali effetti sul loro benessere e su quello delle persone che assistono è facile immaginare.

Il welfare invisibile costruito dalle famiglie italiane mediante l'assunzione di aiuti esterni è una soluzione inevitabilmente precaria a un problema strutturale: alle famiglie si chiede di più di quanto sono in grado di dare. La pandemia lo ha reso più evidente. Per contro, le molte vittime registrate nelle RSA hanno drammaticamente scoperchiato la fragilità della principale alternativa alla soluzione privatizzata dell'assistenza a domicilio, in carenza di soluzioni miste e intermedie. Il più probabile effetto, almeno nel breve periodo, sarà un rafforzamento del "welfare invisibile" delle assistenti

\footnotetext{
${ }^{5}$ Si veda per esempio, nell'ampia letteratura sul tema: Catanzaro e Colombo, 2009; Degiuli, 2016; Sarti, 2011.
} 
familiari, dette badanti, salvo immaginare un tracollo dell'occupazione e dei redditi delle famiglie italiane interessate.

La pubblicistica ha segnalato altresì alcuni effetti positivi, che andranno peraltro verificati più compiutamente nel tempo: collaboratrici familiari non residenti, dotate di permesso di soggiorno e occupate, ma senza contratto, sono state assunte regolarmente, perché altrimenti non avrebbero potuto raggiungere le abitazioni delle famiglie per cui lavorano. Inoltre, l'esplosione del settore delle consegne a domicilio ha offerto opportunità di lavoro a giovani rifugiati africani che non hanno esitato a esporsi a rischi di contagio per trovare un impiego.

La pandemia ha avuto anche un altro effetto, quello di rendere visibili almeno una parte dei lavoratori giustamente definiti “essenziali”, impegnati ad assicurare servizi di vitale importanza per la sopravvivenza del resto della società. Lavoratori spesso umili, malpagati, dall'occupazione precaria se non addirittura irregolare.

I riflettori però non si sono accesi compiutamente sulle origini di questi lavoratori: su quanto cioè tra i lavoratori essenziali incida la componente di origine immigrata. Se complessivamente gli immigrati rappresentano il 10,6\% dell'occupazione regolare del nostro paese (in cifre, 2,45 milioni), proprio nei settori cruciali per il funzionamento quotidiano della società e nei lavori manuali che li sostengono il loro lavoro è ancora più determinante. L'agricoltura è il caso più noto: 17,9\%, senza contare l'occupazione non dichiarata. Allo stesso livello i servizi alberghieri. Ma il dato s'impenna in quelli che l'ISTAT definisce "servizi collettivi e personali": 36,6\% (Ministero del Lavoro, 2019). Troviamo qui il fenomeno già ricordato delle assistenti familiari, ma anche altre categorie non adeguatamente riconosciute: in molte regioni, per esempio, gli addetti alle mansioni ausiliarie della sanità e dell'assistenza residenziale. Sono stati giustamente ricordati i medici in prima linea (19.000 quelli di origine straniera, secondo l'associazione che li rappresenta), spesso gli infermieri (in Lombardia, uno su tre è immigrato), ma se gli ospedali e le RSA funzionano è anche grazie al lavoro semi-nascosto di questi operatori di base, che pure si sono esposti al rischio di contagio per attendere ai loro compiti. Pulizie, magazzini, servizi di recapito sono altri settori ad elevata incidenza di lavoro immigrato: di tutti abbiamo scoperto la necessità, la scarsa visibilità pubblica, le modeste ricompense. Non sempre l'origine di chi li svolge.

Negli Stati Uniti, un rapporto del Center for Migration Studies di New York uscito proprio il $1^{\circ}$ maggio ha reso noto che gli immigrati stranieri forniscono 19,8 milioni di lavoratori ai settori strutturalmente essenziali, concentrati proprio negli Stati più colpiti dalla pandemia. Sono per esempio il $16 \%$ dei lavoratori dei servizi sanitari a livello nazionale, ma raggiungono il 33\% nello Stato di New York e il 32\% in California. "Nel mezzo della pandemia e nei luoghi in cui sono più necessari, gli immigrati stanno lavorando per fermare la diffusione del COVID-19 e per sostenere $i$ loro concittadini statunitensi, spesso con grande rischio personale - ha dichiarato Donald Kerwin, direttore esecutivo del Centro. Questi stessi lavoratori saranno essenziali per la ripresa economica. Meritano il nostro sostegno e la nostra gratitudine"

Questo riconoscimento scaturito da circostanze drammatiche ha finalmente accresciuto la consapevolezza di un fenomeno in corso da anni a livello mondiale: l'importazione di personale sanitario dal Sud verso il Nord del mondo; e per quanto riguarda l'Europa, dall'Est verso l'Ovest. Filippine e India sono i maggiori fornitori a livello mondiale, ma anche l'Africa fornisce personale sanitario al resto del mondo (Williams, 2019). A rendere più complessa questa geografia del lavoro

\footnotetext{
${ }^{6} \mathrm{https}: / /$ mailchi.mp/cmsny/us-essential-workers-05-01-20?e=88b1455b7d. Scaricato dal sito il 25 maggio 2020.
} 
di cura transnazionale concorre anche il fatto che la regione del Golfo e l'Estremo Oriente sono diventati poli di attrazione sempre più rilevanti. Statistiche non recentissime mostrano che nel Regno Unito, primo importatore europeo, circa un quarto degli infermieri sono stranieri, ma in certe aree superano il 50\%, mentre la Germania sta ricorrendo sempre più ai suoi vicini dell'Europa Orientale (Quartararo, 2019). Comprendendo l'assistenza agli anziani, le stime dell'OIL (Organizzazione internazionale del Lavoro) parlano di circa 100 milioni di immigrati occupati nel mondo in attività di cura (Faist, 2019).

I paesi più sviluppati reclutano all'estero professionisti del settore, formati in paesi più poveri con notevoli costi a carico del welfare locale, tanto che da anni si parla di "pesca di frodo" di medici, infermieri e tecnici da parte dei paesi più forti a danno dei più deboli, benché parzialmente compensata dall'invio di rimesse in direzione opposta (Williams 2019). Questo crescente squilibrio si configura come una delle più serie sfide della "questione sociale transnazionale" posta in rilievo da Faist (2019).

\section{Il ritorno delle sanatorie: un classico delle politiche migratorie italiane (e non solo)}

Nel corso del blocco delle attività considerazioni in parte simili si sono faticosamente fatte strada anche nel dibattito italiano, traducendosi in una serie di appelli per la regolarizzazione degli immigrati privi di validi titoli di soggiorno, tra cui va ricordato un ordine del giorno votato all'unanimità dall'assemblea del CNEL, quindi ufficialmente condiviso dalle rappresentanze dei datori di lavoro? Il caso che ha trainato l'apertura si è verificato nel settore agricolo, con la ministra Bellanova in prima fila, fino al punto di minacciare le dimissioni. Un simile ruolo del dicastero dell'agricoltura su una materia del genere è forse un unicum nella storia delle politiche migratorie italiane ed europee. Una novità è stata anche la richiesta ufficiale delle organizzazioni del settore agricolo di una misura di emersione: forse per la prima volta, almeno in Italia, i datori di lavoro si sono pronunciati così apertamente per una sanatoria degli immigrati in condizione irregolare. Di solito in passato, quanto meno a livello nazionale, avevano preferito aspettare in silenzio e raccogliere i benefici delle sanatorie (colpo di spugna sulle assunzioni di persone prive di documenti validi), senza esporsi politicamente su una materia scottante.

Le sanatorie, come è abbastanza noto, hanno costituito di fatto il pilastro delle politiche migratorie italiane (Pastore, Salis e Villosio, 2013): sette le principali, dal 1986 al 2012, a cui si aggiunge ora, dopo una pausa inusuale di ben otto anni, quella del maggio 2020 (tab.1).

Tabella 1. Immigrati beneficiari delle sanatorie italiane

\begin{tabular}{|l|l|}
\hline Anno & Numero beneficiari \\
\hline 1986 & 118.700 \\
\hline 1990 & 217.626 \\
\hline 1995 & 244.492 \\
\hline 1998 & 217.124 \\
\hline 2002 & 646.829 \\
\hline 2009 & 295.112 \\
\hline 2012 & 134.576 \\
\hline
\end{tabular}

\footnotetext{
${ }^{7}$ https://www.cnel.it/Documenti/Studi-indagini-ricerche-e-ordini-del-giorno. Scaricato il 27 maggio 2019.
} 
Mentre in una prima fase l'immigrazione emersa con le sanatorie veniva prevalentemente dall'Africa (58\% dei beneficiari nella sanatoria del 1990, legata al nome del ministro Martelli), nelle ultime edizioni la provenienza europea ha prevalso (Di Pasquale e Tronchin, 2020): anche grazie alle sanatorie l'immigrazione in Italia da anni è prevalentemente femminile, europea, di tradizione culturale cristiana, contrariamente alle rappresentazioni tuttora correnti.

Le manovre di emersione italiane da diverse edizioni sono state collegate a un rapporto di lavoro instaurato di fatto e congegnate come concessioni nei confronti dei datori di lavoro. Questi ultimi assurgono al ruolo di arbitri del destino dei loro dipendenti privi di un permesso di soggiorno valido. L'emersione non è un diritto soggettivo della persona straniera soggiornante in Italia magari da anni, bensì una facoltà che lo Stato attribuisce ai datori di lavoro.

Si è trattato di un espediente, non solo italiano, per sanare una contraddizione tra politica, economia e società. Lo Stato ha di fatto periodicamente riconosciuto che il mercato, nelle sue pratiche di reclutamento di manodopera, aveva largamente superato i prudenti limiti delle quote d'ingresso, non accontentandosi neppure dei lavoratori con cittadinanza dell'Unione Europea. Piegandosi ai dati di realtà, i governi si riparavano dietro le spalle dei datori di lavoro, affermando in sostanza: noi non avremmo lasciato nuovi immigrati rimanere e lavorare, ma, se proprio li volete, pagate una cifra forfetaria per i contributi evasi e metteteli in regola.

Il compromesso è ancora più interessante perché non coinvolge soltanto imprenditori convenzionali, ma anche semplici cittadini. Anch'essi diventano soggetti attivi del mercato del lavoro, quando assumono collaboratrici e assistenti familiari per le loro necessità domestiche. In un singolare gioco delle parti, molti cittadini che protestano ad alta voce contro l'immigrazione e magari votano per $i$ partiti che vorrebbero contrastarla, si attivano periodicamente per far emergere alla regolarità le singole persone che lavorano presso di loro. Le tre sanatorie del 2002, 2009 e 2012 hanno visto i datori di lavoro familiari in prima linea: nel 2002 (Bossi-Fini) più o meno allo stesso livello delle aziende; nel 2009 il provvedimento, con Maroni ministro degli Interni, era a loro riservato; nel 2012 non lo era, ma di fatto le famiglie hanno monopolizzato i rapporti di lavoro instaurati.

Un altro dato appariscente è stato il protagonismo dei governi di centro-destra e di ministri dall'arcigna retorica anti-immigrati nelle grandi sanatorie della prima decade di questo secolo: avendo rassicurato gli elettori sul loro inattaccabile curriculum di difensori dei confini e della sicurezza nazionale, magari rafforzato da appositi decreti, e non avendo nemici a destra pronti ad attaccarli per sconsiderate aperture all'invasione, disponevano dei margini di manovra per rispondere con pragmatismo alle esigenze del mercato del lavoro. Di solito si sono trovati più in difficoltà sulla materia i governi di centro-sinistra, che hanno fatto ricorso preferibilmente, con l'ultimo governo Prodi, a decreti flussi di grandi proporzioni e ripetuti per due anni consecutivi. Mentre l'aggancio con i fabbisogni del mercato del lavoro sfumava (che senso poteva mai avere far entrare dopo due anni dei lavoratori richiesti tanto tempo prima?), la funzione latente dei decreti-flussi come sanatorie implicite di rapporti di lavoro già instaurati, con persone già arrivate e inserite al lavoro, diventava uno dei segreti più noti del funzionamento delle politiche migratorie italiane.

Un altro elemento di rilievo riguarda il ruolo dei datori di lavoro come intermediari dell'inserimento e della regolarizzazione degli immigrati. Una cospicua letteratura da anni insiste sulla complicità inconfessata tra governi e datori di lavoro: la chiusura dei confini sarebbe in realtà apparente, e servirebbe a fornire al mercato manodopera priva di diritti e del tutto flessibile (per es.: Calavita, 
2005; De Genova, 2002; Ruhs e Anderson, 2010). I governi lucidamente avrebbero introdotto le misure di contrasto dell'immigrazione per rispondere alle aspettative degli imprenditori, affamati di manodopera sfruttabile, in regime quasi schiavile.

Che gli immigrati privi di status legale trovino lavoro e vengano sfruttati è un dato di fatto, e non solo nel settore agricolo di cui più spesso si discute. L'idea però che dietro le quinte esista una strategia occulta è contraddetta dalla disponibilità a favorire l'emersione e ad assumere regolarmente, che così tanti datori di lavoro hanno dimostrato in occasione delle sanatorie (e dei decreti-flussi) (Ambrosini, 2016). Sul versante opposto, i governi si sono mostrati sempre più determinati a contrastare l'ingresso e il soggiorno degli immigrati indesiderati, apparendo più sensibili alle pulsioni xenofobe che agli interessi degli attori economici. Politica e mercato sono spesso in conflitto in materia d'immigrazione, e l'argomento degli accordi occulti o della tolleranza inconfessata ha perso quota con il progressivo indurimento delle misure governative e intergovernative (Sciortino, 2011), come quelle concordate a livello di Unione Europea. La lotta al terrorismo ha fornito argomenti e consenso alla linea della chiusura, così come la difesa di sistemi di welfare in affanno, indebolendo la tolleranza verso l'immigrazione irregolare. Le politiche trumpiane sono l'esempio più notevole di una volontà politica che guarda al consenso degli elettori, pressoché incurante dei fabbisogni del mercato del lavoro.

Occorre allora domandarsi: quali ragioni inducono i datori di lavoro a regolarizzare lavoratori che potrebbero continuare a impiegare in nero, godendo di un'impunità raramente scalfita dai blandi controlli ispettivi delle autorità? ${ }^{8}$ Credo che i fattori in gioco siano molteplici. Il primo riguarda i costi di socializzazione e formazione: rifiutare la regolarizzazione significa aumentare i rischi di defezione e quindi di perdita degli investimenti formativi. La flessibilità di molte forme di lavoro regolare non è poi molto minore di quella del lavoro irregolare. Molte mansioni, anche esecutive, richiedono inoltre applicazione costante, dedizione, senso di responsabilità. Questo è particolarmente vero nell'ambito familiare, dove alle lavoratrici immigrate è affidato il benessere di persone fragili e il coinvolgimento personale è cruciale per assicurare i livelli di assistenza desiderati. Difficile immaginare di mantenerli intatti dopo aver respinto una richiesta di emersione. Sempre nell'ambito familiare, ma anche in molti contesti di piccolissima impresa, sul lavoro si instaurano delle relazioni interpersonali che possono assumere una densità emotiva difficilmente aggirabile. Non dev'essere facile lavorare fianco a fianco tutti i giorni e guardare negli occhi una persona a cui non si è accordata la facoltà di accedere a uno status legale, con i diritti connessi. Non si può escludere infine il timore di sanzioni, sebbene remote e improbabili: in caso per esempio di un infortunio sul lavoro difficilmente spiegabile.

\section{Un'emersione contrastata e parziale}

La misura di emersione del decreto-rilancio del maggio 2020 è maturata in un clima di acceso contrasto politico, anche all'interno della stessa maggioranza di governo.

La proposta, come già accennato, ha preso le mosse da una scomoda ma impellente verità: la previsione di mancanza di braccia per raccogliere la frutta e gli ortaggi dell'agricoltura. I 18.000 immigrati stagionali autorizzati negli scorsi anni dai decreti-flussi, anche sotto la gestione Salvini, nel 2020 non sono arrivati. Gli immigrati disoccupati ma residenti in altre regioni non si potevano spostare, né probabilmente volevano farlo. Gli italiani coperti dal reddito di cittadinanza non sono

\footnotetext{
${ }^{8}$ Per una discussione di questo aspetto cruciale, cfr. Chauvin e Garcés-Mascareñas, 2020
} 
mai apparsi ansiosi di prendere la via dei campi. Non la prendevano prima del 2018, sarebbe stato difficile spingerli a farlo al tempo della pandemia.

Quindi dove non sono arrivati gli afflati umanitari, ha trovato un certo spazio la considerazione degli interessi economici: quei prodotti agricoli andavano raccolti da qualcuno, per remunerare i produttori e per rifornire le nostre tavole. Da parte sindacale, si inseriva l'auspicio che il possesso di un titolo di soggiorno avrebbe consentito di rivendicare contratti di lavoro e diritti.

Vari appelli, come quello dell'ASGI (Associazione Studi Giuridici sull'Immigrazione), quello dei sindacati confederali e quello proposto dagli economisti Tito Boeri e Leonardo Becchetti hanno allargato la prospettiva: non solo i lavoratori agricoli, ma pure gli altri, anch'essi più che mai esposti di questi tempi a precarietà e sfruttamento. La condizione delle assistenti e collaboratrici familiari, come ho già ricordato, è stata quella salita alla ribalta con maggiore evidenza, anche alla luce dei risultati delle precedenti sanatorie.

Si inseriva poi, sebbene cautamente, una visione più ampia: in tempi di pandemia, sarebbe stato saggio estendere l'emersione a tutti gli stranieri che si trovano sul territorio nazionale, in modo da poterne monitorare le condizioni di salute e fornire assistenza in caso di bisogno. Per legge infatti gli immigrati irregolari hanno diritto soltanto alle cure mediche "urgenti e necessarie".

Questa posizione è stata assunta in modo esplicito dal CNEL, come ho già accennato. Le parti sociali che lo compongono, insieme a esperti e organizzazioni del terzo settore, hanno convenuto sulla proposta di una misura di emersione universalistica, al fine di tutelare le condizioni di salute dell'intera popolazione, italiana e immigrata. La circolazione di persone non controllate, per definizione indotte a sottrarsi al rapporto con le istituzioni pubbliche, era indicata come un rischio da scongiurare.

Le obiezioni non sono mancate. Il principio di legalità, secondo i critici, ne sarebbe uscito offuscato. La presenza sul territorio nazionale di circa 400.000 immigrati ufficialmente disoccupati (Ministero del Lavoro, 2019) avrebbe sconsigliato di regolarizzarne altri. Gli italiani beneficiari di reddito di cittadinanza avrebbero potuto rendersi utili lavorando in agricoltura.

Gestire il problema dell'immigrazione irregolare in sintonia con le leggi vigenti avrebbe tuttavia richiesto espulsioni di massa. Ma queste non sono mai riuscite, neppure in tempi più normali: nel 2017 quelle effettivamente attuate sono state 6.514; nel 2018, con Salvini alla guida del Ministero degli Interni, si sono fermate a 6.820 (Paletti e Russo, 2019: 171). Diversi fattori ne ostacolano l'attuazione: difficoltà e resistenze nell'identificazione (Ellermann, 2010); mancanza di accordi con i paesi di origine e problemi di effettiva applicazione; scarsi finanziamenti per i ritorni volontari assistiti, e modesto impegno nel promuoverli; ingenti costi e oneri organizzativi del trattenimento e del rimpatrio degli immigrati irregolari. È improbabile che si possa attuarne di più ora, con gli aeroporti chiusi e i paesi di origine ancora meno disponibili ad accoglierli, ammesso che si riesca a individuarli.

La clemenza appariva dunque la migliore delle soluzioni possibili, nello stesso interesse del nostro paese. Ma la contrarietà del M5S ha reso molto più controverso e tortuoso il percorso decisionale. Dopo molto travaglio ed estenuanti discussioni, una misura di emersione a favore degli immigrati privi di uno status legale ha trovato posto nel decreto rilancio. Le lacrime della ministra Belladonna, sua principale promotrice, hanno suggellato un risultato fino all'ultimo in bilico. Strappare un provvedimento del genere a un parlamento largamente ostile a ogni apertura a favore degli immigrati era forse insperabile. 
Certo, la logica della norma è piuttosto obliqua. Dove non è arrivata la tutela dei diritti umani, sono arrivati gli ortaggi da raccogliere nei campi. Dove non ha fatto breccia la protezione dal contagio di persone prive di accesso ai servizi, con conseguenza anche per la nostra salute, è passata una stentata accoglienza delle braccia necessarie a certi settori. Dove si poteva approfittare dell'occasione per risanare i guasti dei decreti sicurezza, e insieme di un mercato nero del lavoro senza garanzie, si è preferito discriminare tra un'occupazione e l'altra, tra uno sfruttamento e l'altro.

Di fatto, il provvedimento ricalca le orme delle politiche migratorie all'italiana, ricordate nelle pagine precedenti. La politica di regolazione dell'immigrazione ancora una volta ha seguito il mercato: una volta che i datori di lavoro, in questo caso solo di alcuni settori, avranno deciso di assumere dei lavoratori stranieri, governo e parlamento glielo concederanno, sia pure dopo polemiche e contorsioni. In negativo questa volta entra in ballo appunto la discriminazione settoriale: saranno salvati i lavoratori di agricoltura, zootecnia, pesca, servizi domestici e assistenziali presso le famiglie. Era già successo d'altronde qualcosa del genere col decreto Maroni del 2009, riservato a colf e assistenti familiari, dette badanti. Porte chiuse per gli altri. Lavorare in un cantiere edile, in un ristorante $\mathrm{o}$ in un'impresa di pulizie non comporta possibilità di emersione.

A lenire il danno compare la possibilità di assunzione futura: se l'attuale manovale o l'addetta alle pulizie, o anche il disoccupato, troveranno ora un datore di lavoro dei settori "giusti”, potranno essere regolarizzati. Poi tra qualche mese, grazie alla possibilità di conversione del contratto, avranno eventualmente la possibilità di transitare verso altre occupazioni. Magari verso quella che già stavano svolgendo in precedenza.

Non è difficile prevedere le conseguenze, già d'altronde riscontrate nelle precedenti sanatorie: si farà avanti una schiera di datori di lavoro di comodo, pronti a offrire contratti di assunzione fittizi dietro compenso. Gli immigrati, per i quali l'emersione alla legalità è un bisogno assoluto, rischiano di cadere in un'altra forma di sfruttamento.

Il provvedimento rimane quindi lontano dalle aspettative dei promotori. Ma era difficile aspettarsi di più da chi poco più di un anno fa votava i decreti di Salvini e perseguitava le ONG che salvavano le persone in mare. C'è voluto lo choc della pandemia per provocare almeno un parziale ripensamento.

\section{Conclusioni. Una nuova sfida per la società civile}

La pandemia da COVID-19 ha investito la popolazione immigrata in Italia, con conseguenze disparate e anche contraddittorie. Per alcune, segnatamente sul piano economico e occupazionale, solo la fase post-confinamento mostrerà le effettive dimensioni.

Certamente il confinamento ha aggravato le condizioni di vita delle fasce sociali più esposte alla precarietà, impegnate in lavori occasionali o irregolari, o in stentate pratiche di sopravvivenza. In tempi difficili, chi vive alla giornata paga di solito i prezzi più alti.

Il brusco peggioramento delle condizioni economiche degli immigrati ha inoltre ripercussioni transnazionali, sotto forma di calo delle rimesse che consentono alle famiglie nei paesi di origine di condurre una vita dignitosa, dando altresì ossigeno alle economie locali.

Il punto si collega alla questione sociale transnazionale (Faist, 2019): la pandemia rischia di inasprire le disuguaglianze globali, colpendo con effetti ancora più gravi i contesti già fragili di molti paesi del Sud del mondo. I loro stessi sistemi di protezione sociale sono indeboliti dal drenaggio di personale sanitario qualificato da parte dei paesi più sviluppati, a loro volta dipendenti nei confronti dei paesi 
più deboli sotto il profilo della fornitura delle risorse necessarie per funzioni cruciali come la cura della salute (Williams, 2019).

Tra le implicazioni potenzialmente positive dell'emergenza, risalta il fatto che i lavoratori definiti “essenziali", a partire da quelli del settore sanitario, sono usciti dall'ombra, acquisendo un'inedita popolarità. Anche se non sempre l'apporto degli immigrati è stato adeguatamente riconosciuto, la scoperta che la vita quotidiana della società si basa sul lavoro di operatori poco considerati, spesso malpagati, in molti casi con occupazioni instabili, non potrà essere facilmente rimossa.

Nella scoperta del nesso tra immigrazione e lavori essenziali s'inscrive il provvedimento di parziale emersione degli immigrati in condizione irregolare: il progresso più rilevante generato in Italia dalla pandemia e dal confinamento nella gestione politica dei processi migratori.

L'impatto effettivo dell'opportunità di regolarizzazione è tuttavia incerto. Il governo, nella relazione tecnica al Decreto rilancio, avanza una stima di 220.000 beneficiari. Molto dipenderà in realtà dalla mobilitazione degli attori pro-immigrati della società civile che in questa difficile stagione hanno cercato in vario modo di contrastare l'ondata di ostilità contro i nuovi arrivati e di compensare la reticenza delle istituzioni pubbliche nella salvaguardia dei diritti umani (Ambrosini, 2014).

Si apre quindi una fase fluida e incerta, in cui sarà decisivo il sostegno agli immigrati che chiederanno ai datori di lavoro di essere regolarizzati. Diverse azioni saranno probabilmente promosse. Aiutare entrambe le parti a produrre la documentazione necessaria, indirizzandole agli sportelli sindacali o di organizzazioni riconosciute e affidabili. Sensibilizzare altri datori di lavoro alla possibilità di favorire l'emersione di persone che comunque sono qui e ci rimarranno. Estendere la conoscenza delle opportunità di emersione. Contrastare la compravendita di falsi contratti. Sostenere le campagne per un lavoro agricolo decente e regolare, ma adottare la stessa logica anche nelle famiglie.

Il protagonismo della società civile, così importante in molti passaggi critici della ricezione dei rifugiati e degli immigrati nel nostro paese, è chiamato a misurarsi ora con la prova di questa contrastata opportunità di emersione.

\section{Bibliografia}

Ambrosini M. (2014). Networking, protesta, advocacy, aiuto. La società civile italiana e gli immigrati. Mondi migranti, 8, 3: 201-222.

Ambrosini M. (2016). From 'Illegality' to Tolerance and Beyond: Irregular Immigration as a Selective and Dynamic Process. International Migration 54, 2: 144-159.

Ambrosini M. (2018). Le migrazioni oltre le polemiche e i luoghi comuni: dieci anni di Mondi Migranti. Mondi Migranti, 12, 1: 7-26.

Ambrosini M. (2019). Famiglie nonostante. Come gli affetti sfidano i confini. Bologna: Il Mulino

Ambrosini M. (2020). L'invasione immaginaria. L'immigrazione oltre i luoghi comuni. Roma-Bari: Laterza.

Calavita, K. (2005). Immigrants at the margins. Law, race and exclusion in Southern Europe. Cambridge: Cambridge University Press.

Catanzaro, R. e Colombo, A. (a cura di), Badanti \& Co. Il lavoro domestico straniero in Italia, Bologna, Il Mulino

Chauvin S. e Garcés-Mascareñas B. 2020. Contradictions in the Moral Economy of Migrant Irregularity. In: Spencer S. e Triandafillydou A. (a cura di) Migrants with Irregular Status in Europe. Evolving Conceptual and Policy Challenges. Amsterdam: Amsterdam University Press, pp. 33-49.

Colasanti R., Geraci S., Pittau F. (a cura di) (1991). Immigrati e salute; paure, miti e verità. Roma: Edizioni Lavoro. 
Colombo E. (2020). Sociologia delle relazioni interculturali. Roma: Carocci

De Genova, N. (2002). Migrant 'illegality' and deportability in everyday life. Annual Review of Anthropology, 31, 1: 419-447.

Degiuli F. (2016). Caring for a living. Migrant women, aging citizens and Italian families. Oxford: Oxford University Press.

Di Pasquale E. e Tronchin C. (2020). Immigrazione vantaggi e svantaggi di una sanatoria. Lavoce.info 21.02.2020. Scaricato dal sito https://www.lavoce.info/archives/63662/immigrazione-vantaggi-e-svantaggidi-una-nuova-sanatoria/

Ellermann, A. (2010). Undocumented Migrants and Resistance in the Liberal State. Politics \& Society, 38, 3: 408-429.

Faist T. (2019). The Transnationalized Social Question. Migration and the Politics of Social Inequalities in the Twenty-First Century. Oxford: Oxford University Press.

Kerwin D., Nicholson M., Alulema D., and Warren R. (2020). US Foreign-Born Essential Workers by Status and State, and the Global Pandemic. Center for Migration Studies of New York report. Scaricato dal sito https://cmsny.org/publications/us-essential-workers/ il 25 maggio 2020.

Ministero del Lavoro e delle Politiche sociali. (2019). Gli stranieri nel mercato del lavoro in Italia. Nono rapporto annuale. Roma: Ministero del Lavoro e delle Politiche sociali.

Molli S.M. (2018). Gli stranieri stanno bene. Salute e integrazione degli immigrati. Milano: ITL.

Paletti F. e Russo F. (2019). Il contrasto dell'immigrazione irregolare. In IDOS (2019). Dossier statistico immigrazione. Roma: Idos, pp. 167-172.

Pastore F., Salis E. e Villosio C. (2013). L'Italia e l'immigrazione low cost: fine di un ciclo? Mondi Migranti, 7, 1: 151-171

Peloso F. (2020). I paesi più poveri rischiano il collasso senza le rimesse degli emigrati. Internazionale, 19 maggio 2020. Scaricato dal sito https:/www.internazionale.it/opinione/francesco-peloso/2020/05/19/rimesseemigrati-collasso il 27 maggio 2020.

Primon J.L. 2019. Il razzismo, un oggetto di ricerca? Il caso della Francia post-coloniale. Mondi Migranti 13, 2: 39-59.

Quartararo C.I. (2019).'Right(S) Opportunities'.The Case of Overseas Nurses and Re-Qualified Health Auxiliary Professionals in Milan. Tesi di dottorato, Graduate School in Social and Political Sciences, Università di Milano.

Rebughini P. e Leonini L. (2019). Guardando oltre l'eurocentrismo: differenze culturali e paradossi della crisi europea. Mondi Migranti, 13, 2: 9-20

Ruhs, M. e Anderson B. (2010). Semi-Compliance and Illegality in Migrant Labor Markets: An Analysis of Migrants, Employers and the State in the UK. Population Space and Place, 16, 3:195 - 211.

Sarti, R. (a cura di) (2011). Lavoro domestico e di cura: quali diritti? Roma: Ediesse.

Sciortino G. (2011). Regolarizzazioni: introduzione. Mondi Migranti, 5, 1: 27-36.

Triandafyllidou A. (2020). Spaces of Solidarity and Spaces of Exception at the times of Covid-19. International Migration. Published on line in early view. https://oi.org/10.1111/imig.12719.

Williams F. (2019). A Multidimensional Analysis of Migration and Care Work. Mondi migranti, 13, 3: 9-24. Zini A. (2019). Pilastro sociale e motore economico: il lavoro domestico nell'Europa e nell'Italia del futuro. In IDOS (2019). Dossier statistico immigrazione. Roma: Idos, pp. 296-298. 


\section{L'immigrazione al tempo della pandemia: nuove difficoltà, scoperte impreviste, opportunità insperate}

L'articolo apre una riflessione sull'impatto della pandemia da COVID-19 sulla condizione delle popolazioni immigrate in Italia. Prende in considerazione l'impoverimento dei lavoratori che hanno perso il lavoro e altre fonti di sostentamento; l'ondata di ostilità nei confronti delle persone di origine cinese e la chiusura nei confronti dei richiedenti asilo salvati in mare; le difficoltà delle lavoratrici domestiche e assistenziali. Per contro, il confinamento ha posto in rilievo il ruolo dei lavoratori che assicurano servizi essenziali, specialmente nel settore sanitario: tra questi, anche se non sempre riconosciute, molte persone di origine immigrata.

Nella seconda parte l'articolo tratta la conseguenza più importante della pandemia nell'ambito migratorio: l'apertura di una seppur parziale e contrastata possibilità di emersione per i lavoratori agricoli e occupati presso le famiglie. Questa novità viene collocata nel solco delle politiche migratorie italiane e discussa nelle sue opportunità e contraddizioni

Parole chiave:

immigrazione; COVID-19; sanità; lavoratori essenziali; regolarizzazione

\section{Abstract (english)}

\section{Immigration at the time of pandemic: new difficulties, unexpected discoveries, unhoped-for opportunities}

The article opens a reflection about the impact of the COVID-19 pandemic on the condition of immigrant population in Italy. It considers the impoverishment of workers who have lost their employment and other sources of income; the wave of hostility against people of Chinese origin and the closure of entries to asylum seekers rescued at sea; difficulties of domestic and care workers. On the other side, the lockdown has also highlighted the role of workers providing essential services, especially in the health sector: among them many with an immigrant background, even if not always recognized.

In the second part, the article treats the most relevant consequence of the pandemic in the field of immigration: the opening of a partial and contentious window of regularization in the case of workers employed in agriculture and in households. This new fact is placed in the tradition of Italian immigration policies and discussed in its opportunities and contradictions.

Key words:

Immigration; COVID-19; health system; essential workers; regularization 